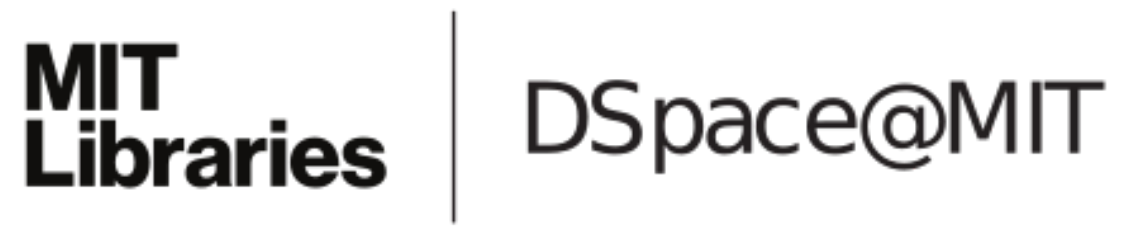

\author{
MIT Open Access Articles
}

Gravitational wave detection with advanced ground based detectors

The MIT Faculty has made this article openly available. Please share how this access benefits you. Your story matters.

Citation: Evans, M. “Gravitational Wave Detection with Advanced Ground Based Detectors.” General Relativity and Gravitation 46.10 (2014): n. pag.

As Published: http://dx.doi.org/10.1007/s10714-014-1778-z

Publisher: Springer US

Persistent URL: http://hdl.handle.net/1721.1/103521

Version: Author's final manuscript: final author's manuscript post peer review, without publisher's formatting or copy editing

Terms of use: Creative Commons Attribution-Noncommercial-Share Alike 


\title{
Gravitational Wave Detection with Advanced Ground Based Detectors
}

\author{
M. Evans on behalf of the LIGO Scientific \\ Collaboration, the Virgo Collaboration, \\ and the KAGRA Collaboration
}

the date of receipt and acceptance should be inserted later

\begin{abstract}
There are great expectations for the world-wide network of gravitational wave detectors currently under construction in United States, Europe and Japan. These multi-kilometer interferometers are seeking to make the first direct detection of gravitational waves and initiate a new era in astronomy and astrophysics in which we can listen to black holes, neutron stars and other enigmatic inhabitants of the local universe. Advanced detectors will be approximately 10 times more sensitive than the initial detectors, which were operational until 2011. As the detection volume for a given source is proportional to the cube of the sensitivity, detection rates will increase by roughly a factor of one thousand; rate estimates suggest a most probable level of tens of events per year once detectors reach design sensitivity. In this paper we give a general overview of the advanced detectors which will start producing astrophysically interesting data in 2015, and we discuss prospects for beginning the observation of the gravitational wave Universe within this decade.
\end{abstract}

\section{Introduction}

Gravitational wave detection offers a deep probe into contorted regions of space-time governed by General Relativity in the strong field regime, at a level where the remnants of giant stars become physics laboratories impossible to create on Earth.

The basic principle behind gravitational wave detection on Earth is the fact that the passage of a gravitational wave perturbs the relative distance between inertial masses. While truly inertial masses cannot be had for long on earth, suspended masses provide a good approximation in the plane perpendicular to Earth's gravitational pull. Thus, a set of suspended mirrors arranged in a

Massachusetts Institute of technology

185 Albany Street, NW 22-275

E-mail: mevans@ligo.mit.edu 
Michelson interferometer configuration provides a coupling from gravitational waves to electro-magnetic waves in which the gravitational wave's properties are imprinted in the interference pattern of the light extracted at the Michelson's anti-symmetric port. Given a Michelson interferometer with arm length $L$, and a gravitational wave with amplitude $h$ passing by the detector, the induced change of the interferometer length $\Delta L$ is given by:

$$
\Delta L=\frac{h L}{2}
$$

The measurement of $\Delta L$ is the essential basis of operation of all gravitational wave interferometers. General Relativity predicts extremely faint gravitational waves amplitudes, with $h \sim 10^{-22}$ for a solar-mass source at a few tens of Mpc.

Unfortunately, $10^{-22}$ is a very small number and detecting a fractional change of this magnitude is highly challenging. Even with multi-kilometer interferometers, the expected displacement is $\Delta L \sim 10^{-18} \mathrm{~m}$, one thousand times smaller than the radius of a proton. Achieving the sensitivity necessary to detect gravitational waves has been the goal of many scientists world-wide since large scale interferometer were proposed for the first time in Europe [1, 2] and United States [3] more than 20 years ago [4].

The initial gravitational wave detectors LIGO, VIRGO and GEO, operated from 2005 to 2011 carrying out a series of data taking runs of increasing duration and sensitivity. More than a year of coincident data among at least two detectors did not show any trace of gravitational waves [5], consistent with calculations of rates using current knowledge of source populations [6].

An enormous effort in instrument science research and technology development enabled the design of the $2^{\text {nd }}$ generation of gravitational wave detectors, the most sensitive position meters ever built, aiming for a factor of 10 increase in sensitivity over the initial detectors. These interferometers, known as the "Advanced Detectors", are currently under constructions in United States (two Advanced LIGO detectors [7]), Italy (Advanced Virgo [8]), Japan (KAGRA $[9]$ ). There is also the possiblility of adding a third Advanced LIGO detector in India, for which construction has yet to begin.

Because the detection volume for a given source is proportional to the cube of the sensitivity, expected detection rates are approximately a factor of one thousand higher than those of the initial detectors. Predictions of event rates cover a range of values, with 10's of events per year possible [10]. A century after Einstein conceived their existence, the era of gravitational wave detection in which the Universe becomes audible is finally upon us.

\section{Astrophysical reach}

The sensitivity of gravitational wave detectors is typically expressed in terms of their astrophysical reach, i.e. how far in the Universe they are able to observe. This metric depends entirely on the source considered; the canonical 


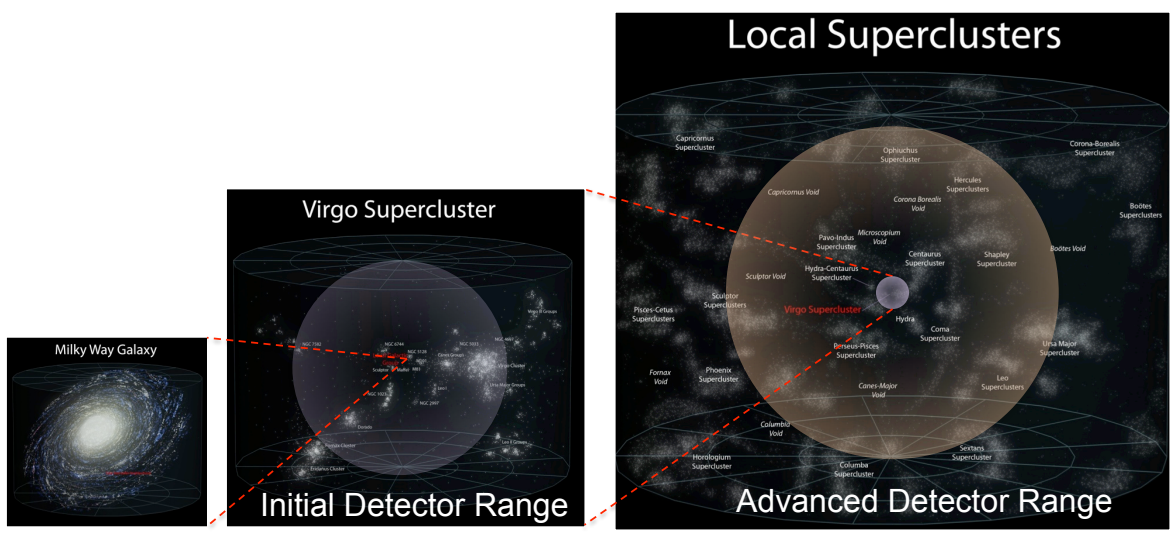

Fig. 1 Observable Universe for initial and advanced detectors.

source used as a reference is a 1.4 -1.4 $M_{\odot}$ binary neutron star system, detectable with a signal-to-noise-ratio (SNR) of 8 . For this canonical source, the advanced gravitational waves interferometers under constructions have a design sensitivity of 130 to $200 \mathrm{Mpc}$. The Advanced LIGO interferometers have the highest design sensitivity, 200 Mpc [11], while the Advanced Virgo design sensitivity is around $130 \mathrm{Mpc}$ [12]. The interferometer arm length is the primary cause of the difference in sensitivity between the two detectors, which are otherwise conceptually very similar. Advanced Virgo is $3 \mathrm{~km}$ long, while the Advanced LIGO detectors are $4 \mathrm{~km}$ long, thus making the Advanced Virgo sensitivity (roughly) $3 / 4$ of the Advanced LIGO one. The $3 \mathrm{~km}$ long detector KAGRA will incorporate new technologies, such as cryogenic cooling of the test masses, and its design sensitivity is $176 \mathrm{Mpc}[13]$.

Given the density of compact binary coalescences in our Universe, it is possible to estimate how many events the advanced detectors will observe, given their sensitivity and the duration of operation. However, because of the uncertainties in the astrophysical rate predictions for compact binary coalescences, the uncertainties on the rate estimate can reach up to 2 orders of magnitudes in each direction from the most likely prediction. We refer to [6] for a discussion of the expected rates for advanced detectors.

A simple rate estimate can be done in the following way. A realistic number for binary neutron stars coalescences is one per $\mathrm{Mpc}^{3}$ every million years

$$
R_{r e}=\frac{10^{-6}}{\mathrm{Mpc}^{3} \cdot \text { year }} .
$$

A detector with a range $R$ will be able to observe a sphere in the Universe of volume $\frac{4}{3} \pi R^{3}$. This is roughly $3 \times 10^{7} \mathrm{Mpc}^{3}$ for the advanced detectors, and $3 \times 10^{4} \mathrm{Mpc}^{3}$ for the initial detectors (see figure 1). Given an observation time of 1 year, the expected number of events for an advanced detector is

$$
N_{r e}=R_{R e} \times\left(\frac{4}{3} \pi R^{3}\right) \times(1 \text { year }) \sim 30,
$$


or a few per month, while for an initial detector $N_{r e} \sim 0.03$

\section{The path to Advanced detectors}

The $2^{\text {nd }}$ generation of gravitational wave detectors share a broadly common path to improved performance. They all depart from their predecessors by attacking the three fundamental noise sources discussed in the next section; quantum noise is reduced with increased laser power, thermal noise with improved suspensions and optics, and seismic noise with high performance isolation systems.

In addition to the changes aimed at reducing these fundamental noises, all of the advanced detectors benefit from the community's experience with technical noises and operability issues encountered in the $1^{\text {st }}$ generation of detectors. The results of these changes are evident in new designs which promise improved stability and mitigation of technical noises which troubled earlier detectors (e.g., reduction of noise from scattered light with extensive baffling, and isolation from acoustic noise through in-vacuum photo-detection) $[14,15]$. The new designs have also produced new technical noises and challenges, along with a myriad of mitigation techniques [16-20]. While these technical feats are likely to prove critical to the success of the $2^{\text {nd }}$ generation, they are beyond the scope of this article.

\subsection{The fundamentals}

The fundamental noises are what limit a gravitational wave detector's sensitivity after the careful removed innumerable technical noises (themselves the target of extended research during long periods of detector commissioning). These noises are called fundamental in the sense that they can not be overcome without significant changes to the detector hardware, not in the sense that they cannot be changed without altering the laws of nature [21].

Figure 2 shows, as an example, the fundamental noise sources which are expected to limit the Advanced LIGO sensitivity: quantum noise, thermal noise, and seismic noise. Very similar curves describe Advanced Virgo and KAGRA.

\subsubsection{Quantum Noise}

Among the designers of the $1^{\text {st }}$ generation of detectors, no one spoke of "quantum noise", but rather of "shot noise" which arises from the random arrival times of photons on a photo-detector $[22,23]$. The statistics of this process give rise to a noise level which is determined by the power on the photo-detector $P_{\mathrm{LO}}$ by the relation $N=\sqrt{2 P_{\mathrm{LO}} h \nu}$, where $h \nu$ is the energy of a single photon. The signal produced by an interferometer is proportional to $\sqrt{P_{\mathrm{LO}}} \times \sqrt{P}$ where $P$ is the light power stored inside the interferometer modulated by a 


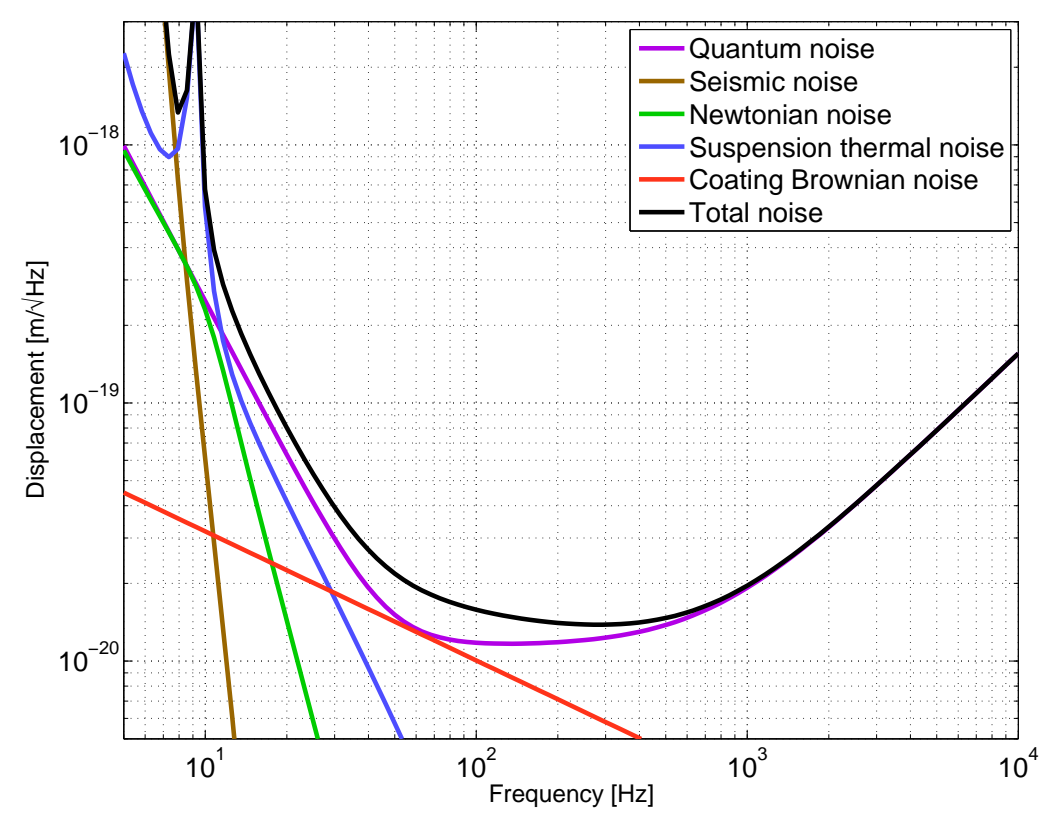

Fig. 2 Fundamental noise sources limiting the sensitivity of an interferometric gravitational wave detector [21]. The dominant quantum noise mechanism is shot noise above $60 \mathrm{~Hz}$, and radiation pressure noise below that frequency. To achieve this level of sensitivity, nearly $1 \mathrm{MW}$ of optical power must circulate in the detector. Thermal noise enters mostly through the suspensions below $30 \mathrm{~Hz}$, while the coatings dominate in most of the band. Environmental noises are well isolated, such that the dominant mechanism is though the direct Newtonian coupling to the test-masses.

passing gravitational wave. The signal to noise ratio increases therefore with increasing the interferometer power as $\sqrt{P}$.

As the field began to contemplate higher power designs for $2^{\text {nd }}$ generation detectors it became clear that this simplistic approach to the quantum mechanics of light would not be sufficient. In particular, the quantization of light also leads to a random force being applied to the optics in the interferometer known as "radiation pressure noise" (RPN). A conceptually simple picture of $\mathrm{RPN}$ comes again from the random arrival times of photons, but this time on the mirrors of the interferometer. As the mirrors reflect these photons, they reverse the photons' momentum and are thus kicked in the opposite direction. The sum of many random impulses becomes a force noise on the interferometer optics which cannot be distinguished from a gravitational wave signal. Increasing power means more photons and thus more RPN, going with $\sqrt{P}$. Since this is a force noise which induces a displacement of the optics, the signal it produces also increase with $P$, so that the signal to noise ratio decreases with increasing power as $1 / \sqrt{P}$.

This understanding is, however, lacking in that it fails to reveal the intimate connection between shot noise and RPN which arises from their com- 
mon origin. A complete understanding of quantum noise in optical systems with radiation pressure gave designers of $2^{\text {nd }}$ generation detectors the tools they needed to optimize the optical layout and laser power [24,25]. The result of such an optimization can be seen in figure 2 as the purple "Quantum Noise" curve, which is dominantly RPN below $60 \mathrm{~Hz}$ and shot noise above that frequency.

\subsubsection{Thermal Noise}

Another fundamental noise source in gravitational wave detectors and other precision optical measurements arises from the thermal motion of the atoms in the most sensitive parts of the instrument. The two most important sources of thermal noise in advanced detectors are the optical coatings (coating thermal noise), and the suspension elements which support the optics against the force of gravity (suspension thermal noise).

As compared with the $1^{\text {st }}$ generation detectors, great improvements have been made in suspension thermal noise owing to the move away from metal wires as support elements. Essentially all of the advanced detectors use fused silica fibers to support the interferometer optics, as this material has low mechanical losses and high tensile strength [26]. The thermal noise from these suspensions is expected to be more than an order of magnitude lower than that of the wire suspensions used in previous detectors. Figure 2 shows that the impact of suspension thermal noise on Advanced LIGO is essentially to define the low-frequency end of the detection band.

Reduced suspension thermal noise brought coating thermal noise to the forefront as a limiting noise source of the $2^{\text {nd }}$ generation detectors $[27,28]$. The extremely stringent optical requirements placed on these coatings (e.g., less than 1ppm absorption to avoid thermal problems due to the $1 \mathrm{MW}$ of circulating power) make optimization of the mechanical properties to reduce thermal noise very challenging. In fact, less than a factor of 2 improvement has been made since the initial detectors, and much of the improvement attained came simply from increasing the beam size on the optics (since the noise amplitude scales with beam radius). Coating thermal noise, also shown in figure 2, limits the sensitivity of the advanced detectors in the region most critical to binary neutron star and black hole detection, and has proven to be the least mobile of the fundamental noises.

KAGRA, unique among advanced detectors in its use of cryogenics, will reduce the impact of thermal noise by using a crystalline test mass material cooled to $20 \mathrm{~K}[29]$.

\subsubsection{Seismic Noise}

The las tand possibly least fundamental of the "fundamental noises" are the vibrations which enter a gravitational wave detector from the external environment. These disturbances, collectively referred to as "seismic noise", generally arrive at the detector in the form of a seismic wave and have a variety of 


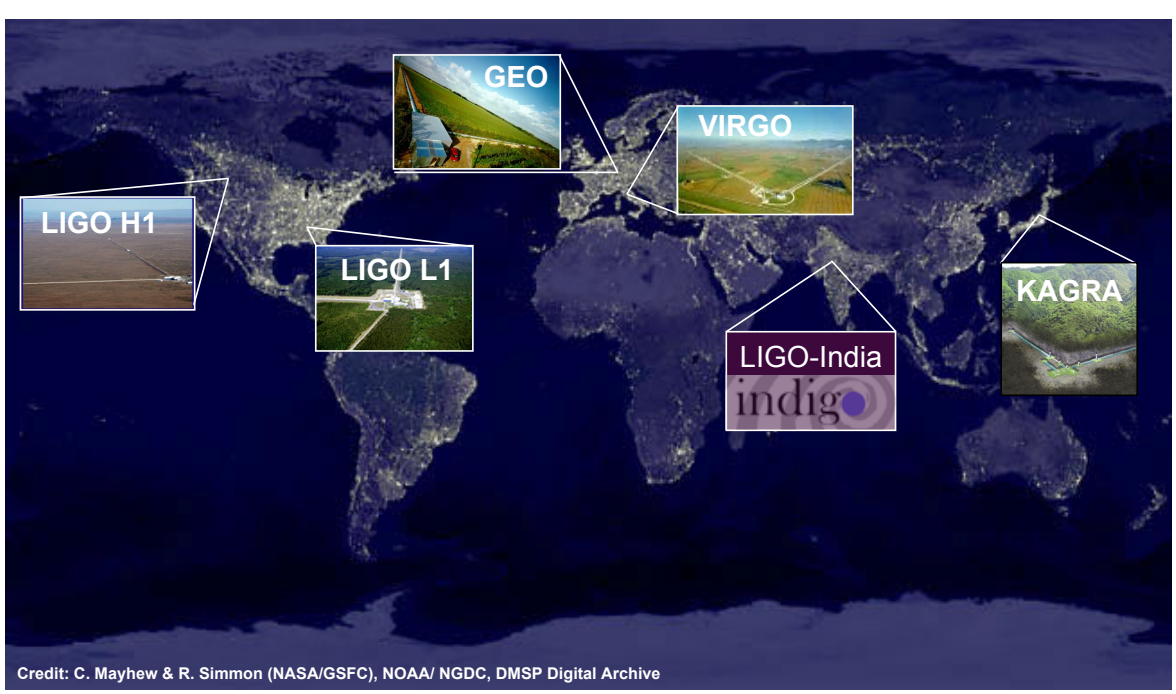

Fig. 3 World-wide network of advanced gravitational wave detectors.

origins: from ocean waves crashing on a far away beach to passing trains, and from wind buffeting the local terrain to machinery which keeps the detector running. Essentially anything which moves can be a noise source and sophisticated isolation systems are needed to avoid contaminating the gravitational wave signal.

Isolation systems in the $2^{\text {nd }}$ generation detectors generally have one or more "active stages", with acceleration sensors and feedback loops, and four or more passive stages which use the isolation provided by mechanical inertia. In the end, it is the rejection of high frequency motion by the inertia of large masses that keeps the vibrations of the world from masking a gravitational wave signal.

These systems are so effective that the dominant coupling of motion from the environment in the sensitive band of the detector is the only one that cannot be shielded in any way: the Newtonian gravitational coupling of external moving masses to the interferometer's test-masses, known as "Newtonian Noise" (see figure 2) [30]. While larger than seismic noise at all frequencies above $10 \mathrm{~Hz}$, Newtonian noise is not expected to be a dominant noise source in the advanced detectors, though it presents a significant problem for future generations.

\section{Overview of the World-Wide Network}

The gravitational wave network in the Advanced Detector Era is shown in figure 3. It is comprised of the two $4 \mathrm{~km}$ long Advanced LIGO detectors in United States, the $3 \mathrm{~km}$ long Advanced Virgo detector in Italy, the $3 \mathrm{~km}$ long KAGRA detector in Japan, and the 600m GEO detector in Germany. At the 
time of this writing, it appears likely that a third LIGO detector will be built in India by the end of the decade.

While the $2^{\text {nd }}$ generation detectors share many common features, as discussed in section 3 , they also have differences which we will be briefly describe here. For more details about the current status of each detector, please refer to the section "Progress and Challenges in Advanced Ground Based Gravitational Wave Detectors" in these proceedings.

\subsection{Advanced LIGO}

The Advanced LIGO detectors are located in Hanford Washington and in Livingston Louisiana, and typically referred to as $\mathrm{H} 1$ and L1, are separated by about 3000 miles. Installation of $2^{\text {nd }}$ generation hardware began at the LIGO observatories in 2010.

The Hanford Observatory can accommodate two co-located interferometers which share the same vacuum tubes, and this feature was used in time of the the initial detectors to house a second detector, known as H2. The original Advanced LIGO plan was continue with $\mathrm{H} 1$ and $\mathrm{H} 2$, however, at the beginning of the installation phase it became clear that moving $\mathrm{H} 2$ to a new site outside of the US could provide a remarkable scientific benefit. Now this plan is coming to fruition with a potential move to India, and all the components of the H2

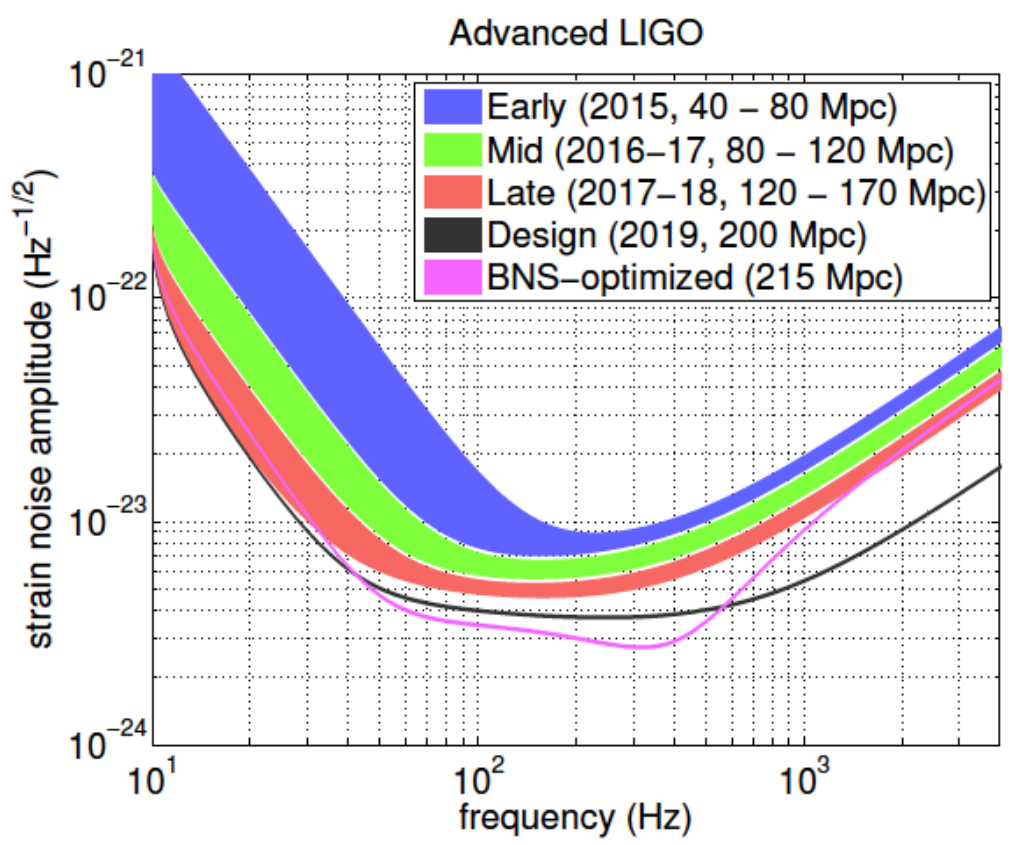

Fig. 4 Plausible progression of aLIGO sensitivity curves over the next decade [10]. 
interferometer are being put in storage, to be shipped with the beginning of the LIGO-India project.

When operating at full power, the Advanced LIGO arm cavities will store $800 \mathrm{~kW}$ of laser power, or about 20 times more than initial LIGO. The Advanced LIGO test masses are $40 \mathrm{~kg}, 34 \mathrm{~cm}$ diameter fused silica mirrors which represent the state of the art in civilian optics technology in terms of absorption losses and surface uniformity. The most significant upgrade for LIGO are the new active seismic isolation systems. Multi-stage active isolators [31] combined with a quadruple stage of pendula [26,32], are able to essentially eliminate the seismic noise transferred to the main optics, thus extending the detection band down to $10 \mathrm{~Hz}$.

The Advanced LIGO detectors will finish their installation phase in 2014, and start observing in 2015. A plausible observing scenario is shown in figure 4 , which shows a progressive increase which reaches the design sensitivity of $200 \mathrm{Mpc}$ at the end of this decade. The envisioned strategy is to perform observing runs, from a few months to one year, after reaching sensitivity milestones of roughly $50 \mathrm{Mpc}, 100 \mathrm{Mpc}$, and $150 \mathrm{Mpc}[10]$.

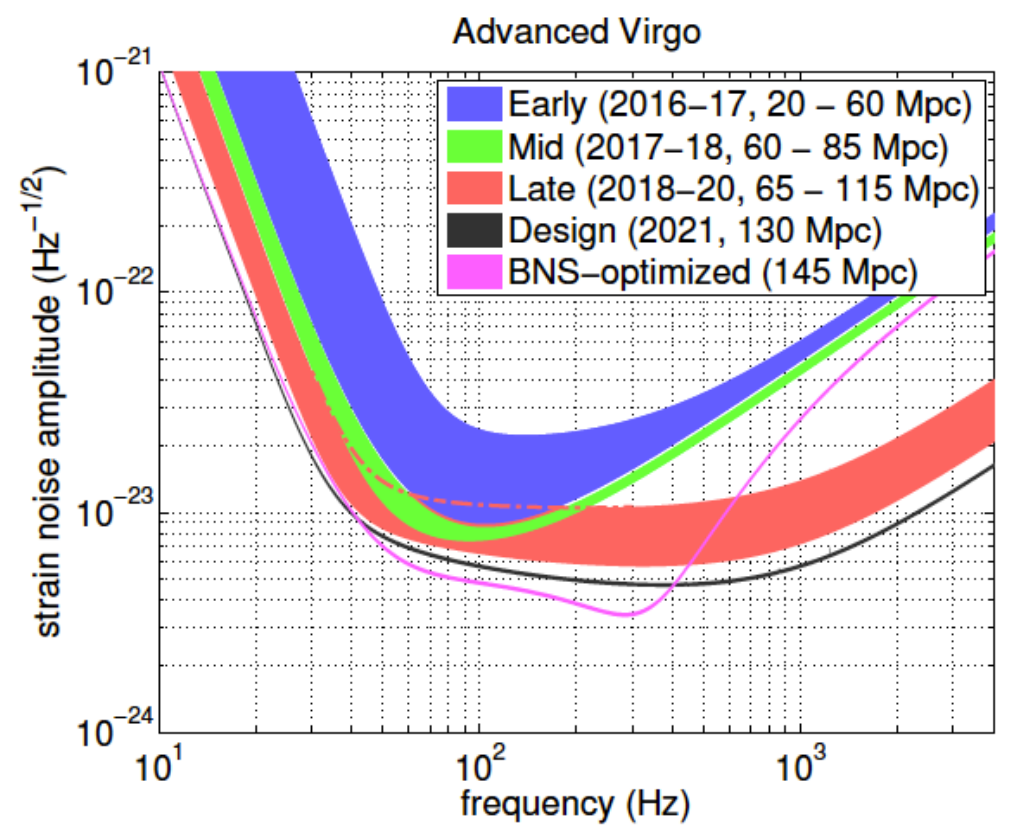

Fig. 5 Plausible progression of Advanced VIRGO sensitivity curves over the next decade [10]. 


\subsection{Advanced Virgo}

Advanced Virgo is currently under construction at the European Gravitationalwave Observatory site near Pisa, Italy, which hosted the Virgo detector until 2011. Because high performance suspensions were already employed in Virgo (the super-attenuator [33]), Advanced Virgo will be the advanced detector most similar to its predecessor. A factor of 10 better sensitivity with respect to the initial Virgo detector is achieved by increasing the circulating power in the interferometer and employing larger and heavier state of the art optics.

Figure 4.2 shows a plausible observing scenario for Advanced Virgo. Advanced Virgo will join Advanced LIGO observations in 2016, and it will follow a path similar to the Advanced LIGO one. The sensitivity will be progressively increased until reaching design sensitivity of $130 \mathrm{Mpc}$ in about 5 years, while joining the Advanced LIGO observing runs [10].

\subsection{KAGRA}

The Japanese advanced detector KAGRA is the most recent addition to the network and is significantly different from Advanced LIGO and Advanced Virgo. It has the unique features of being built underground and is designed to operate at cryogenic temperatures, thus becoming the pathfinder of technologies for future generation detectors.

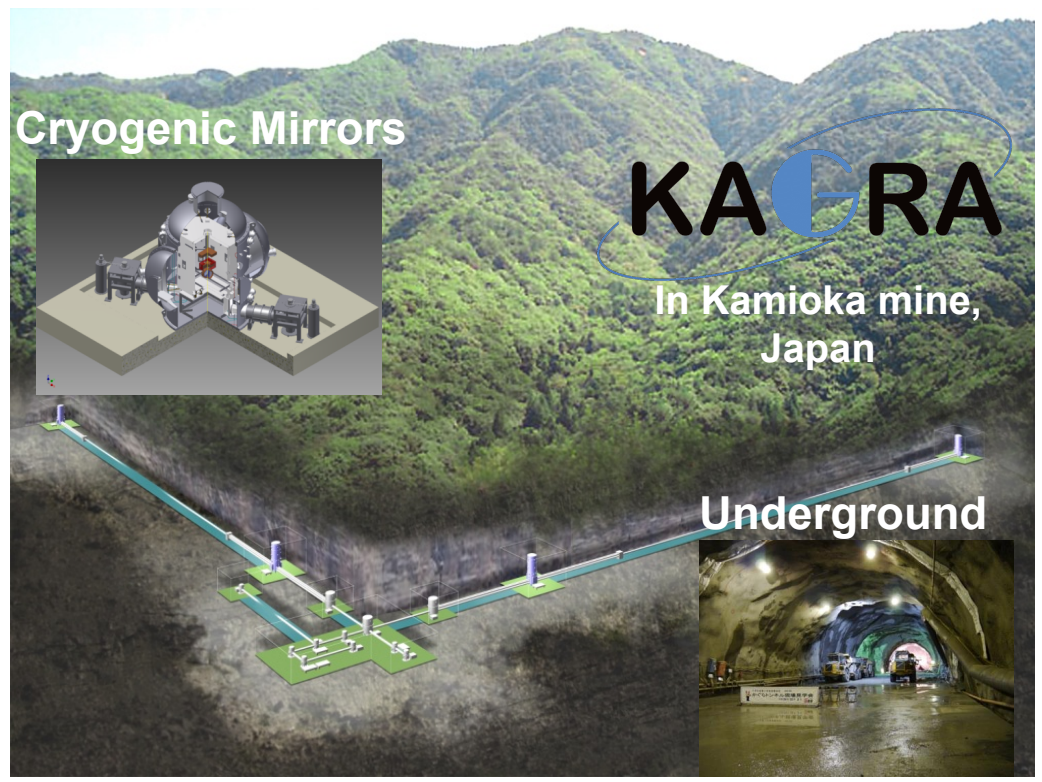

Fig. 6 Main features of the KAGRA detector: it is cryogenic, and built underground. 
The excavation of the KAGRA tunnels was completed mid-2014. According to the current plan, KAGRA will join the advanced detector network in 2017, then progressively improve the detector performance until reaching the design sensitivity of about $176 \mathrm{Mpc}$.

\subsection{GEO600}

The $600 \mathrm{~m}$ long GEO interferometer is located close to Hannover, in Germany. Because it is significantly shorter than the other detectors, GEO has an astrophysical interesting sensitivity only at frequencies above $1 \mathrm{kHz}$. On the other hand, it has the remarkable feature of having operated for the last decade with what are now considered "advanced technologies".

At the time of this writing, GEO is the only gravitational wave detector in operation, and thus it provides coverage while waiting for the advanced detectors to come on-line. By its nature GEO targets specific sources of gravitational waves, such as supernovae explosions, which could produce a gravitational wave signal in the high frequency region.

Continuing its tradition of blazing trails for future detectors, GEO is already using technologies which will appear in $3^{\text {rd }}$ generation detectors, such as squeezed light, thereby playing a critical role in the R\&D effort [34].

\section{Future Detectors}

Even as the advanced detectors are coming on-line, the instrumental community is looking ahead to what will come next. In all likelihood, detection rates with the $2^{\text {nd }}$ generation detectors will not be more than a few per month and at best a handful of high signal-to-noise ratio events will be accumulated over the life of the detector $[6,10]$. While successful detection of gravitational wave is a great achievement by any measure, there is a clear motivation to improve detector sensitivity as quickly as possible.

Significant design effort has already gone into planning future gravitational wave detectors $[21,35,36]$. Three major phases can be identified in the progress beyond the advanced detectors: short term upgrades to the current detectors which may occur in the next 3-5 years and aim to improve sensitivity by as much as a factor of 2 without major disruption to data taking, medium term overhauls intended to maximize sensitivity in the current facilities, and finally the construction of new facilities with detectors designed to achieve a factor of 10 improvement relative to the advanced detectors.

Two areas of active research which may result in technology applicable in the short term are squeezed light for reduction of quantum noise, and lownoise optical coatings. The first of these has been demonstrated to reduce shot noise in $1^{\text {st }}$ generation detectors $[37,38]$, and is considered a relatively mature technology. Application of squeezed light to $2^{\text {nd }}$ generation detectors is somewhat complicated by the radiation pressure effects (see section 3.1.1), 
and research into the experimental techniques required for producing squeezed light compatible with advanced interferometers is well underway $[39,40]$.

An advanced detector which incorporates squeezed light will inevitably become limited by coating thermal noise and require improved coatings to realize the goal of doubling the instrument's sensitivity. Recent developments in crystal coating make such an improvement plausible [41], though this new technology has yet to be demonstrated on the $30 \mathrm{~cm}$ scale optics required by gravitational wave detectors. Should these or other low noise coatings prove to be workable on large scale optics, their installation in the current detectors could, in combination with squeezed light, increase the detection rate of astrophysical sources by as much as a factor of 10 .

In the medium term, more disruptive changes have the potential to further improve detector sensitivity while still working inside the vacuum envelope of the current facilities. Areas of active research include cryogenics, moving to crystalline optics for improved thermal conductivity and greater power handling (e.g., sapphire and silicon), and a shift in laser wavelength to $1550 \mathrm{~nm}$ (required for silicon optics). Several of these features are already being incorporated in the new KAGRA detector in Japan (see section 4.3), while others are being developed at the laboratory scale.

To attain a factor of 10 improvement in sensitivity, and thus a factor of 1000 increase in event rate relative to the $2^{\text {nd }}$ generation, will almost certainly require that we go beyond the current facilities. An inescapable feature of gravitational wave detection is that longer detectors have greater strain sensitivity for a given displacement sensitivity, as seen in equation 1. Assuming no major paradigm shift in gravitational wave detector technology, future facilities will need to be a factor of 3 or more longer than current detectors. Another major opportunity afforded by the construction of new facilities is the move to a triangular configuration in which three interferometers, each rotated by $60^{\circ}$ relative to the other two, share the same vacuum system and enable the detection of both polarizations of the gravitational wave radiation from astrophysical sources [35].

\section{Conclusion}

This is an exciting time in gravitational wave physics. The world-wide network of ground based interferometers currently under construction expects to detect up to tens of gravitational wave detections every year. The direct detection of gravitational waves will give us a completely new way to listen to the Universe. This tool will give us access to events and regimes not accessible to electromagnetic observations, and add invaluable information to events which can be observed with both gravitational and electromagnetic radiation.

The advanced detectors have all started their construction phase, and they will begin producing astrophysically interesting data in the coming years: the Advanced LIGO detectors in US are planned to come on line in 2015, followed 
by Advanced Virgo in 2016 and KAGRA in 2017. A third Advanced LIGO detector could also be built in India by the end of this decade.

These new detectors will be the most sensitive position meters ever built, relying on the incredible progress in instrument science and technology which made them possible. In parallel, the GEO detector and laboratory efforts world wide are studying ways of making the advanced detectors more sensitive by pushing the state of the art, with the goal of extending even further the observable gravitational wave Universe.

\section{References}

1. The Virgo Collaboration: Virgo: Proposal for the construction of a large interferometric detector of Gravitational waves (1989)

2. J. Hough, et al: Proposal for a Joint German-British Interferometric Gravitational Wave Detector, Report MPQ 147, Max Planck Institute for Quantum Optics, Garching, Germany (1989)

3. LIGO Project: A Laser Interferometer Gravitational-Wave Observatory (LIGO), Proposal to the National science Foundation (1989)

4. R. Weiss: Electronically Coupled Broadband Gravitational Antenna, Quarterly Progress Report, Research Laboratory of Electronics (MIT) No. 105, p.54. (1972)

5. M. Bizouard: Observational results from the LIGO and Virgo detectors, these proceedings (2014)

6. J. Abadie et al.: Predictions for the Rates of Compact Binary Coalescences Observable by Gravitational-wave Detectors, Class. Quantum Grav. 27173001 (2010)

7. G. Harry (for the LIGO Scientific Collaboration): Class. Quantum Grav. 27084006 (2010)

8. The Virgo Collaboration: Advanced Virgo Baseline Design VIR-0027A-09 (2009) at https://pub3.ego-gw.it/itf/tds/

9. K. Somiya: Class. Quantum Grav. $29 \quad 124007 \quad$ (2012) doi:10.1088/02649381/29/12/124007

10. J. Aasi et al.: Prospects for Localization of Gravitational Wave Transients by the Advanced LIGO and Advanced Virgo Observatories, arXiv:1304.0670 (2013)

11. These proceedings "Status of Advanced LIGO" (in Progress and Challenges in Advanced Ground Based Gravitational Wave Detectors)

12. These proceedings "Advanced Virgo" (in Progress and Challenges in Advanced Ground Based Gravitational Wave Detectors)

13. These proceedings "Progress and Challenges of KAGRA" (in Progress and Challenges in Advanced Ground Based Gravitational Wave Detectors)

14. B. Canuel et al.: Displacement noise from back scattering and specular reflection of input optics in advanced gravitational wave detectors, Optics Express, Vol. 21, Issue 9, pp. 10546-10562 (2013)

15. D. Ottaway, P. Fritschel and S. Waldman: Impact of upconverted scattered light on advanced interferometric gravitational wave detectors, Optics Express, Vol. 20, Issue 8, pp. 8329-8336 (2012)

16. M. Evans, L. Barsotti and P. Fritschel: A general approach to optomechanical parametric instabilities. Physics Letters A, 374(4), 665671. (2010) doi:10.1016/j.physleta.2009.11.023

17. R. Dolesi, M. Hueller, D. Nicolodi, D. Tombolato, S. Vitale, P. Wass, et al.: Brownian force noise from molecular collisions and the sensitivity of advanced gravitational wave observatories. Physical Review D, 84(6), 063007. (2011)

18. A. Mullavey, B. Slagmolen, J. Miller, M. Evans, P. Fritschel, D. Sigg, et al.: Arm-length stabilisation for interferometric gravitational-wave detectors using frequency-doubled auxiliary lasers. arXiv:1112.3118. (2011)

19. J. Miller, M. Evans, L. Barsotti, P. Fritschel, M. MacInnis, R. Mittleman, et al.: Damping parametric instabilities in future gravitational wave detectors by means of electrostatic actuators. Physics Letters A, 375(3), 788794. doi:10.1016/j.physleta.2010.12.032 (2011) 
20. J. Driggers, M. Evans, K. Pepper and R. Adhikari: Active noise cancellation in a suspended interferometer. Review of Scientific Instruments, 83(2), 024501. (2012) doi:10.1063/1.3675891

21. R. Adhikari: Gravitational Radiation Detection with Laser Interferometry, arXiv:1305.5188 (2013)

22. W. Edelstein et al.: J. Phys. E: Sci. Instrum. 11710 (1978) doi:10.1088/0022$3735 / 11 / 7 / 030$

23. P. Fritschel, R. Bork, G. Gonzlez, N. Mavalvala, D. Ouimette, H. Rong, et al.: Readout and Control of a Power-Recycled Interferometric Gravitational-Wave Antenna. Applied Optics, 40(28), 49884998. (2001) doi:10.1364/AO.40.004988

24. C. M. Caves: Quantum-Mechanical Radiation-Pressure Fluctuations in an Interferometer, Phys. Rev. Lett. 45, 75 (1980).

25. A. Buonanno and Y. Chen: Quantum noise in second generation, signal-recycled laser interferometric gravitational-wave detectors, Phys. Rev. D 64, 042006 (2001).

26. A. Cumming, A. Bell, L. Barsotti, M. A Barton, G. Cagnoli, D. Cook, L. Cunningham, M. Evans, G. Hammond, G. Harry, A. Heptonstall, J. Hough, R. Jones, R. Kumar, R. Mittleman, N. Robertson, S. Rowan, B. Shapiro, K. Strain, K. Tokmakov, C. Torrie, and A. van Veggel: Design and development of the advanced LIGO monolithic fused silica suspension, Classical and Quantum Gravity, 29(3):035003 (2012)

27. G. Harry, M. Abernathy, A. Becerra-Toledo, H. Armandula, E. Black, K. Dooley, M. Eichenfield, C. Nwabugwu, A. Villar, D. Crooks, G. Cagnoli, J. Hough, C. How, I. MacLaren, P. Murray, S. Reid, S. Rowan, P. Sneddon, M. Fejer, R. Route, S. Penn, P. Ganau, J. Mackowski, C. Michel, L. Pinard, and A. Remillieux: Titania-doped tantala/silica coatings for gravitational-wave detection. Class. Quantum Grav., 24:405-415 (2007)

28. M. Evans, S. Ballmer, M. Fejer, P. Fritschel, G. Harry, and G. Ogin: Thermo-optic noise in coated mirrors for high-precision optical measurements. Physical Review D, 78(10), (2008)

29. T. Uchiyama et al.: Reduction of thermal fluctuations in a cryogenic laser interferometric gravitational wave detector, Phys. Rev. Lett. 108, 141101 (2012)

30. J. Driggers, J. Harms, and R. Adhikari: Subtraction of Newtonian noise using optimized sensor arrays. Physical Review D 86.10102001 (2012)

31. F. Matichard et al.: Prototyping, testing, and performance of the two-stage seismic isolation system for advanced LIGO gravitational wave detectors Proc. ASPE Conf. on Control of Precision Systems pp 7580 (2010)

32. S. Aston et al.: Update on quadruple suspension design for Advanced LIGO, Class.Quant.Grav. 29235004 (2012)

33. Measurements of Superattenuator seismic isolation by Virgo interferometer, Astroparticle Physics, Volume 33, Issue 3 (2010), Pages 182189

34. H. Grote et al.: "First long-term application of squeezed states of light in a gravitationalwave observatory," Phys. Rev. Lett. 110, 181101 (2013).

35. M. Punturo et al.: The Einstein Telescope: a third-generation gravitational wave observatory, Class. Quantum Grav. 27194002 (2010)

36. M. Punturo et al.: Einstein gravitational wave Telescope conceptual design study (2011) at https://tds.ego-gw.it/ql/?c=7954

37. LIGO Scientific Collaboration: A gravitational wave observatory operating beyond the quantum shot-noise limit, Nature Phys. 7 (12) 962-965 (2011).

38. LIGO Scientific Collaboration: Enhanced sensitivity of the LIGO gravitational wave detector by using squeezed states of light, Nature Photon. 7, 613-619 (2013).

39. H. Kimble, Y. Levin, A. Matsko, K. Thorne, and S. Vyatchanin: Conversion of conventional gravitational-wave interferometers into quantum nondemolition interferometers by modifying their input and/or output optics. Phys. Rev. D 65, 022002 (2001).

40. M. Evans et al.: Realistic filter cavities for advanced gravitational wave detectors, Phys. Rev. D 88, 022002 (2013)

41. G. Cole et al.: Tenfold reduction of Brownian noise in high-reflectivity optical coatings, Nature Photonics 7, 644650 (2013) 